\title{
Comunicación
}

\section{Tripanosomiasis canina en Madre de Dios, Perú}

\author{
Canine trypanosomiasis in Madre de Dios, Peru
}

Jesús Rojas Jaimes ${ }^{1,2,5}$, Edwin Tineo Villafuerte ${ }^{3}$, Roberto Lope Huamán ${ }^{4}$

\section{Resumen}

El objetivo del presente estudio fue reportar la presencia de Trypanosoma sp en una muestra de sangre de un perro en Madre de Dios, Perú. El perro era residente del distrito de Las Piedras y al ser atendido en un centro médico veterinario fue reportado con diagnóstico presuntivo de ehrlichiosis. Se tomó una muestra de sangre y el frotis fue coloreado con Wright, donde se observó los tripomastigotes de Trypanosoma sp. No se realizaron cultivos y estudios moleculares debido a que la muestra se contaminó. Este es el primer reporte de tripanosomiasis canina en Madre de Dios.

Palabras clave: tripanosomiasis; perro; Trypanosoma; Madre de Dios

\section{AbStract}

The aim of this study was to report the presence of Trypanosoma sp in a blood smear from a dog in Madre de Dios, Peru. The animal was a resident of the district of Las Piedras and, when treated at a veterinary medical centre, was reported with a presumptive diagnosis of ehrlichiosis. A blood sample was collected, and the smear was stained with Wright, where trypomastigotes of Trypanosoma sp was observed. No cultures and molecular studies were performed because the sample was contaminated. This is the first report of canine trypanosomiasis in Madre de Dios.

Key words: tripanosomiasis; dog; Trypanosoma; Madre de Dios

\footnotetext{
${ }^{1}$ Escuela de Medicina Humana, Universidad Científica del Sur, Lima-Perú

${ }^{2}$ Facultad de Ciencia de la Salud, Universidad Privada del Norte, Lima Perú

${ }^{3}$ Laboratorio Regional de Salud Pública, Madre de Dios, Perú

${ }^{4}$ Clínica Veterinaria HOVET, Madre de Dios, Perú

${ }^{5}$ E-mail:jesus.rojas.jaimes@gmail.com
}

Recibido: 11 de diciembre de 2018

Aceptado para publicación: 11 de julio de 2019 
INTRODUCCIÓN

La enfermedad de Chagas, también conocida como tripanosomiasis, es causada por el parásito Trypanosoma cruzi. Este agente etiológico es trasmitido por insectos de los géneros Triatoma, Rhodnius y Panstrongylus. Esta enfermedad que causa cardiopatías, megaesófago, y megacolon está distribuida en Estados Unidos, México y Centro y Sudamérica (CFSPH, 2009). La mayor cantidad de casos son registrados en ciclos domésticos, aunque también existe un ciclo selvático menos estudiado (CFSPH, 2009). En el Perú, Herrer (1955) reportó los vectores principalmente en las regiones de Moquegua y Arequipa, así como la presencia del agente etiológico en el cuy y el gato.

La tripanosomiasis canina ejerce un factor importante en la trasmisión al humano en el ciclo doméstico, en especial por T. cruzi en donde el perro se comporta como un hospedero y excepcionalmente por $T$. evansi, que es encontrada en perros con marcada parasitemia y sin signos clínicos, comportándose el animal como un reservorio centinela. En estos casos, el perro se infecta por la ingesta de carne y vísceras de animales contaminados (CFSPH, 2009; Desquesnes et al., 2013)

\section{Materiales y Métodos}

En presente reporte se basó en un estudio descriptivo observacional en un perro mitayero (cazador), macho y mestizo de 7 años, del distrito de Las Piedras, localidad de Planchon, en Tampobata, Madre de Dios, Perú (1207'12.95"S, 6904’31.47"O; 267 msnm). El perro fue atendido primariamente en la clínica veterinaria HOVET en la ciudad de Puerto Maldonado, Madre de Dios. El animal clínicamente presentaba fiebre, inapetencia por 15 días y estaba delgado, con presunción diagnóstica de ehrlichiosis. El perro no estuvo fuera del área de Las Piedras en las semanas previas de su ingreso al consultorio.
Se le tomó una muestra de sangre venosa y se realizó un frotis con tinción Wrigth en el laboratorio de la clínica HOVET. La muestra de sangre restante fue guardada en refrigeración por una semana a $8^{\circ} \mathrm{C}$ aproximadamente. Posteriormente se trasladó a Lima sin cadena de frio ( 2 días) en un tubo Vacutaeiner con anticoagulante para el cultivo en medio bifásico a $35^{\circ} \mathrm{C}$ en el laboratorio de microbiología de la Escuela de Medicina Humana de la Universidad Científica del Sur y detección molecular del kinetoplasto en el laboratorio de Tripanosominos de la Universidad Peruana Cayetano Heredia.

\section{Resultados}

Se encontró tripomastigotes en el frotis, observándose el kinetoplasto en la zona posterior del parásito compatible con Trypanosoma sp (Figura 1). La muestra que se trasladó a Lima para el diagnóstico molecular 1legó contaminada por lo que no pudo ser procesada. Adicionalmente, el can y el dueño no aparecieron de nuevo por el lugar de captación.

\section{Discusión}

Según el reporte del Centro Nacional de Epidemiología, Prevención y Control de Enfermedades - CDC (2017), Arequipa es la región con mayor número de casos de tripanosomiasis humana, mientras que en Madre de Dios solo se ha reportado un caso en 2013 y tres casos en 2014 (CDC, 2017). No obstante, no se señala si son casos autóctonos o importados, siendo esto importante ya que Madre de Dios está contigua a la región Arequipa y recibe una gran migración de esta ciudad, en especial por actividades ligadas al comercio y a la minería ilegal.

En este caso, el perro presentaba cuadros clínicos compatibles con tripanosomiasis, siendo importante señalar que los perros presentan cuadros clínicos similares al humano, 


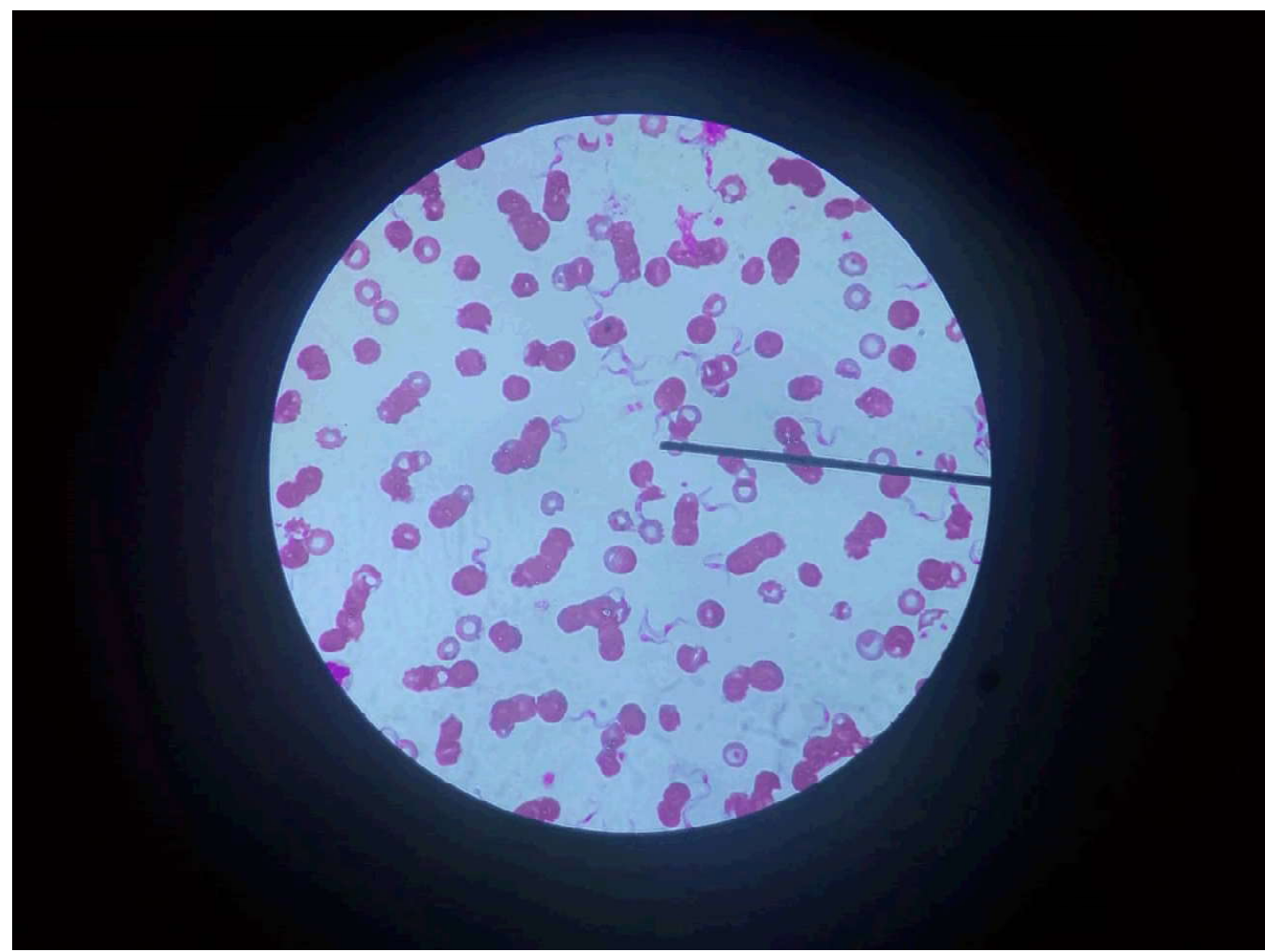

Figura 1. Promastigotes de Trypanosoma sp en un frotis de sangre de perro macho, mestizo, de 7 años, del distrito de Las Piedras, Planchon (Tampobata, Madre de Dios). Tinción Wright. 1000X

tanto en su fase aguda como crónica (Acha y Szyfres, 1986). Cabe mencionar, además, que el perro pudo haberse infectado con un tripanosoma patógeno para el hombre como $T$. cruzi o con T. evansi que infecta al perro, donde se se mantiene asintomático, excepto en algunos casos fatales (Desquesnes et al., 2013).

El perro es un eslabón de suma importancia para la transmisión del parásito al humano, ya que este animal se infecta cuando se encuentra en áreas de trópico (zona selvática) y es contaminado por el vector (Barreto y Ribeiro, 1909). Los perros infectados se vinculan al humano en sus zonas domiciliarias, pudiendo completar la transmisión cuando el vector pica al perro que pre- senta parasitemia sistémica y después contamina al humano (Eloy y Lucheis, 2012).

\section{Conclusiones}

- Se determinó la presencia de Trypanosoma sp en una muestra de sangre de perro en el distrito de las Piedras, Tambopata, Madre de Dios, siendo este animal un centinela que se encuentra en el ciclo de la tripanosomiasis.

- Este sería el primer caso de tripanosomiasis en un perro de Madre de Dios, Perú, por lo que se debe encender un sistema de alarma para la vigilancia de esta enfermedad en la región 


\section{Literatura Citada}

1. Acha P, Szyfres B. 1986. Zoonosis y enfermedades transmitidas comunes al hombre y animales. $2^{\circ}$ ed. Washington DC, USA: OMS. 708 p.

2. Barreto M, Ribeiro M. 1909. Reservatorios Silvestres do Trypanosoma (Schyzotrypanun) cruzi Chagas. Rev Ins Adolfo Lutz 39: 25-36.

3. Centro Nacional de Epidemiologia, Prevención y Control de Enfermedades, Ministerio de Salud- Perú. 2017. Mapa de enfermedad de Chagas por distritos. Perú 2017. [Internet]. Disponible en: http://www.dge.gob.pe/portal/docs/ vigilancia/sala/2017/SE09/chagas.pdf

4. [CFSPH] The Center for Food Security and Public Health. 2009. Chagas disease. [Internet]. Available in:
http://www.cfsph.iastate.edu/FastFacts/ pdfs/chagas_F.pdf

5. Desquesnes M, Holzmuller P, Lai D, Dargantes A, Lun Z, Jittaplapong $S$. 2013. Trypanosoma evansi and surra: a review and perspectives on origin, history, distribution, taxonomy, morpho-logy, hosts, and pathogenic effects. BioMed Res Int 2013: 194176. doi: 10.1155/2013/194176

6. Eloy L, Lucheis S. 2012. Hemoculture and polymerasa chain reaction using primers TCZ1/TCZ2 for the diagnosis for canine and feline tripanosomiasis. Vet Sci 12: ID419378. doi: 10.5402/2012/419378. 419-378

7. Herrer A. 1955. Trypanosomiasis americana en el Perú. I. El insecto vector y los animales que actúan de reservorio de la enfermedad de Chagas en la región sudoccidental. Rev Peru Med Exp Salud Pública 1:23-37. 